OAI-PMH: http://www.indteca.com/ojs/index.php/Revista Scientific/oai

Ensayo Original / Original Essay

\title{
Principios Holísticos y los Docentes en el Contexto de Educación Primaria
}

\author{
Autor: César Enrique López Arrillaga \\ Universidad Latinoamericana y del Caribe, ULAC \\ prof.cesarlopez@gmail.com \\ Caracas, Venezuela \\ https://orcid.org/0000-0002-2926-8508
}

Resumen

El presente ensayo realizará un recorrido teórico de la educación, la escuela y los docentes como elementos del quehacer educativo del contexto de educación primaria relacionados con los principios de la holística postulados por Barrera (2010); y otros referentes teóricos como Freire (1977); Calvo (2015); y Delors (1996), desarrollan el análisis desde la premisa de un contexto holístico para la formación de los estudiantes orientado por la mirada holística en comprender la diversidad, integralidad, unicidad y complejidad de la realidad de los educandos en su proceso de aprendizaje y enseñanza en la educación primaria desde las premisas de la holística en el campo educativo.

Palabras clave: educación; docente; pedagogía; afectividad; docente de escuela primaria.

Cómo citar este ensayo:

López, C. (2019). Principios Holísticos y los Docentes en el Contexto de Educación Primaria. Revista Scientific, 4(14), 368-389, e-ISSN: 2542-2987. Recuperado de: https://doi.org/10.29394/Scientific.issn.2542-2987.2019.4.14.18.368-389

Fecha de Recepción: 15-07-2019
Fecha de Aceptación:

30-09-2019
Fecha de Publicación:

05-11-2019 


\title{
Holistic Principles and Teachers in the Context of Primary Education
}

\begin{abstract}
This essay will conduct a theoretical tour of education, school and teachers as elements of the educational task of the context of primary education related to the principles of holistics postulated by Barrera (2010); and other theoretical references such as Freire (1977); Calvo (2015); and Delors (1996), develop the analysis from the premise of a holistic context for the formation of students guided by the holistic view of understanding the diversity, integrality, uniqueness and complexity of the reality of students in their learning and teaching process in primary education from the premises of holistics in the educational field.
\end{abstract}

Keywords: education; teacher; pedagogy; affectivity; primary school teacher.

Date Received:

15-07-2019
Date Acceptance:

30-09-2019
Date Publication:

05-11-2019 


\section{Introducción}

Al hablar de educación se hace alusión a la transformación de la vida de los seres humanos, en vista que los aprendizajes se construyen de manera colectiva. En tal sentido la educación está orientada a la integración de los miembros de una comunidad de la cual hacen parte, dando lugar a un ambiente de cooperación, lleno de armonía, en el que surgen relaciones interpersonales sanas, que conllevan al cumplimiento de los pilares de la educación sustentados por Delors (1996): quien señala como Aprender a Conocer (lo que supone aprender a aprender); Aprender a Hacer (Adquirir una competencia para capacitarlo para enfrentar situaciones en la vida); Aprender a Vivir (comprensión del otra y aceptación de las diferencias) y Aprender a Ser (que florezca lo mejor de la personalidad en el marco de valores) (pág. 34).

Cabe destacar que se habla de una educación que forma a un individuo de manera natural, que hace parte de un contexto que influye de manera directa, es aquella, sobre la cual muchos autores a lo largo del tiempo al hecho importantes aportaciones, por lo tanto, para Freire (1977a), sostiene que: "la educación verdadera es praxis, reflexión y acción del hombre sobre el mundo para transformarlo" (pág. 135). En efecto, la educación no puede ser un espacio ajeno al contexto y la realidad social sino abierta a recoger las necesidades, inquietudes, sentimientos y vivencias de las comunidades y familias.

Es oportuno destacar, la Organización de las Naciones Unidas para la Educación, Ciencia y Tecnología (UNESCO, 2016), cuando indica que: "la educación transforma vidas y ocupa el centro mismo de la misión de la UNESCO, consistente en consolidar la paz, erradicar la pobreza e impulsar el desarrollo sostenible" (párr. 1). Bajo las premisas anteriores, el presente ensayo versará sobre el recorrido teórico en cuanto a la educación y el docente del siglo XXI y su vinculación desde el contexto de educación primaria con los Principios de la Holística para desarrollo de un proceso de enseñanza y 
aprendizaje holístico desde la diversidad y comprensión los intereses, necesidades del estudiante.

\section{Desarrollo}

\subsection{La Educación y la Escuela como espacios de intercambio de conocimientos.}

La educación es un contexto que permite a los actores educativos transformar sus vidas con herramientas y elementos de desarrollo social, económico y comunitario en su comunidad educativa desde una perspectiva colectiva en sociedad. Es importante tomar en cuenta lo planteado en el Foro Mundial de la Educación realizado por la UNESCO (2015a), al señalar que: "transformar las vidas mediante la educación, reconociendo el importante papel que desempeña la educación como motor principal del desarrollo y para la consecución de los demás ODS propuestos" (pág. 2).

Bajo esta perspectiva del foro, la UNESCO (2015b), plantea: "una educación de calidad y con la mejora de los resultados de aprendizaje, para lo cual es necesario fortalecer los insumos, los procesos y la evaluación de los resultados y los mecanismos para medir los progresos" (pág. 3). Ciertamente, la educación como proceso social se involucra con las características de cada actor educativo y responde al momento histórico que vive la sociedad, significa entonces que desde las políticas públicas de cada país y los esfuerzos colectivos en el fortalecimiento de una educación de calidad para todos. En relación con Camacaro (2017), sugiere:

La educación debe desplegar las posibilidades para los educandos se conviertan en hombres y mujeres responsables en la toma de decisiones, capaces de formarse juicios correctos ante la realidad, respetuosos de los demás, dotados de una sana autoestima y bien posesionados de sus derechos y deberes sociales para el ejercicio de la democracia (pág. 376). 
Sobre la base de lo anteriormente señalado, la educación se fundamente en ampliar sus horizontes para la formación integral y holista en función de transformar la sociedad en el ejercicio de sus deberes y derechos como comunidad, de esta manera consolidar la construcción de personas con principios y valores. En consecuencia, Freire (1977b), sugiere que: "la Educación es un acto de amor, por tanto, un acto de valentía" (pág. 92). Ciertamente se considera la educación un continuo acto de amor en permanente desarrollo que debe orientarse en el cultivo del amor en todos los actores educativos de las escuelas, una comunidad educativa para el amor, que impulse a seres humanos amorosos para una sociedad de amor. Es decir, los docentes en el contexto de educación primaria en la orientación de atender al estudiante desde sus necesidades e intereses de formación bajo la mirada holística.

De allí, los docentes relacionados con la escuela de educación primaria son propicia como un entorno que va forjando a los estudiantes y docentes en sus relaciones con los demás participantes del contexto educativo que influyen en su actuar diario en el presente y futuro, cultivando en ellos los valores, habilidades y destrezas, que lo coadyuvaran en la práctica futura en los ámbitos de la vida de cada educando. Del mismo modo, se manifiesta el rol y la función de la escuela en el contacto educativo, como en efecto Freire (1977c), indica que:

La escuela es la institución donde ocurre la acción profesional del maestro. Esta escuela que ahora conocemos tiene apenas unos doscientos años que se institucionalizó. La institución escolar es la célula básica del sistema educativo, como la familia lo es a la sociedad. Comprender su origen y gestación ayudará a identificar sus enormes potencialidades, así como sus limitaciones frente a una sociedad definida hoy día por el conocimiento y el aprendizaje (pág. 10).

Desde las consideraciones anteriores, es importante señalar lo 
planteado por el Informe Nacional de la República Bolivariana de Venezuela (2004a), referente a Políticas, Programas y Estrategias de la Educación Venezolana, emitido por el Ministerio de Educación y Deportes de Venezuela en el cual se señala la escuela como: "la escuela venezolana tiene un propósito trasformador de la sociedad, pues la práctica educativa es una dimensión necesaria de la práctica social al formar al ciudadano en el ejercicio de sus derechos" (pág. 9).

En consecuencia, el Informe Nacional de la República Bolivariana de Venezuela (2004b), señala que: "la escuela juega un papel preponderante en el resguardo de la identidad nacional al atender la diversidad e incentivar la productividad y el desarrollo sustentable en las comunidades" (pág. 11). Hecha la observación anterior, la escuela posee un rol protagónico en la consolidación de la identidad local y nacional dado su vinculación la familia y comunidad, en el contexto del quehacer educativo.

\subsection{El Docente como guía del proceso educativo.}

Los docentes de educación primaria se desarrollen en la motivación principal en el ejercicio de su rol como mediador de los aprendizajes en pro de una educación integral que responda a las necesidades de cada participante del hecho educativo, En este mismo sentido, para Freire (1993a) "enseñar es una tarea profesional que exige amor, creatividad" (pág. 26). Cabe destacar, los docentes en el nivel primaria son los principales agentes de la transformación del proceso de enseñanza y aprendizaje que propicie la ética y humanización cotidiana en las escuelas. Así mismo, el autor Freire (1993b), sugiere:

El aprendizaje del educador, el enseñar, no se da necesariamente a través de la rectificación de los errores que comete el aprendiz. El aprendizaje del educador al educar se verifica en la medida en que éste, humilde y abierto, se encuentre permanentemente disponible para repensar lo 
pensado, para revisar sus posiciones: se percibe en como busca involucrarse con la curiosidad del alumno (pág. 45).

Desde esta perspectiva, los docentes de educación primaria dentro de su práctica educativa deben incluir todos los factores e indicadores que se relacionan con los estudiantes, en estrecha relación con su entorno social y la comunidad educativa. De igual manera, Freire (1993c), plantea:

El papel del docente es fundamental en el desarrollo personal de sus estudiantes, la transparencia del trato, del contenido de sus lecciones y la actitud frente a lo que enseña, son una garantía para contribuir a la formación de un ser humano que se perciba integralmente y se asuma como gestor social (pág. 24).

Por lo tanto, el docente de educación primaria en el planteamiento de conocer el entorno social de los estudiantes, como premisa vigente para la consolidación de la calidad educativa en el aula y para impulsar un hecho educativo más humano y consciente de las necesidades de formación de sus estudiantes. Así mismo, los docentes en la educación primaria juegan un papel protagónico en el proceso de enseñanza-aprendizaje de los estudiantes en el contexto de la escuela como espacio generador de relaciones interpersonales que se construye en experiencias significativas en la vida colectiva de todos los actores participantes, lo cual consolida la identidad y personalidad de los niños como esencia de actos humanos.

De acuerdo con lo anterior, los docentes en su perspectiva educativa como agente mediador y formador en el proceso de enseñanza y aprendizaje de sus estudiantes, le da la oportunidad de aprender y desaprender en la medida que sea flexible en el intercambio de saberes, conocimientos y experiencias de los participantes en el aula y fuera de ella. Desde la praxis o acción pedagógica de los docentes, cabe agregar lo planteado por Argyris y Schön (1978), quienes sostienen que: 
Los actores sociales construyen una teoría para sus acciones intencionales, y que el comportamiento desplegado, responde, por tanto, a esas construcciones, que en las personas son modelos mentales, mientras que en las organizaciones son los compromisos colectivos expresados en sus propósitos, funciones y actividades organizacionales (pág. 114).

De este modo, la Teoría de la Acción concibe al hombre como un ser vinculado en sus interrelaciones con lo demás, en función a la intencionalidad de modificar su realidad actual, en el marco del entendimiento de las dificultades mediante la construcción del conocimiento colectivo en la educación primaria a través de la acción educativa del docente en las aulas, con la participación activa de los estudiantes, cada actor educativo cumple con funciones y actividades para la consolidación de una formación holística del ser social.

Por ello, el dinamismo de la acción educativa puede proceder de la cultura popular o del ámbito académico. Permitiendo al docente sea más integral, correspondiendo a los intereses de formación de los estudiantes en sus diversas etapas de vida. De allí que, el docente como agente de motivación, es un elemento que impulsa a los estudiantes al descubrimiento de sus sentimientos, ideas y pensamientos para la construcción y consolidación de su propio aprendizaje en la configuración de su personalidad y adquisición de habilidades y destrezas en el marco de una disciplina propia y autónoma. De acuerdo con, McLaren (2005a):

Los maestros necesitan comprender como las experiencias producidas en los diferentes dominios de la vida diaria producen a su vez las distintas voces que los estudiantes emplean para dar significados a sus mundos y por consiguiente, a su existencia en la sociedad (pág. 322).

Por lo tanto, los maestros de la educación primaria mediante la amplitud de su perspectiva y visión holística podrán escuchar y atender las diversas y 
complejas situaciones que presentan los estudiantes en la escuela y aulas de clases. En concordancia con lo anterior, los docentes en la educación primaria en práctica educativa deben alinearse desde una perspectiva amorosa, con una expresión de aceptación y tolerancia de los estudiantes como seres humanos diversos, que permita una convivencia sana en el contexto educativo. Además, McLaren (2005b), expresa que: "la voz del maestro refleja los valores, ideologías y principios estructurales que emplean los maestros para comprender y mediar las historias, las culturas y las subjetividades de sus estudiantes" (pág. 326). El docente desarrolla la capacidad de entendimiento, tolerancia a la diversas de sus estudiantes, rompiendo las barreras de complejos, en función del desarrollo de la personalidad.

Por lo anteriormente descrito se deduce que la problemática del nivel de educación primaria, en cuanto al desempeño del personal docente en relación a la formación holística del estudiante. Así como la integración activa y responsable de los actores del hecho educativo. Ante esta problemática se hace necesario dar respuesta a las aportaciones de los diferentes autores descritos en líneas precedentes. Por lo que es importante que la escuela cuente con la presencia de docentes que den apertura a los cambios que se vienen planteando para el logro de la participación e integración, con visión holística, que sean responsables de las transformaciones que puedan generarse en beneficio de la educación del país en la que se promueva la interacción entre el grupo de informantes clave. Desde esta perspectiva el docente es un factor determinante, que debe estar capacitado para la proyección de la escuela hacia la comunidad, para el logro de una mayor productividad y, por ende, una mayor eficacia.

Desde la experiencia del autor en el ámbito de la escuela primaria, se observa que el funcionamiento de la misma está orientado hacia los procesos de planificación, evaluación, seguimiento y control, no obstante, se deja de lado la vinculación directa con la comunidad. Se identifica la necesidad de una 
escuela que se integre con el resto de las instituciones que funcionan en el entorno, lo que pudiera estar afectando sustantivamente las políticas de implementación del modelo educativo actual y por ende del avance del proceso educativo venezolano.

Se puede apreciar, que los docentes de educación primaria en la actualidad y producto del proceso de postmodernidad y la globalización, lo que han abrumado en su visión y percepción del hecho educativo, siendo más mecanicista y tomar a los estudiantes como recipientes de conocimientos y repetidores de contenidos. Después de las consideraciones anteriores, es importante señalar que los docentes no se encuentran vinculados con los intereses de los educandos, en su práctica educativa es mecánica solo cumplen lo pedagógico, sin embargo, desestiman la integralidad de los estudiantes.

En cuanto al docente como figura central del quehacer educativo en los sistemas de educación relacionado estrechamente con el proceso de enseñanza y aprendizaje de seres humanos para la consolidación de ciudadanos impregnados de valores éticos, sociales y espirituales correlacionados con la integración de la sociedad. En consecuencia, la labor docente considerada como un trabajo, sin embargo, es necesario transcender de la idea laboral, sino visualizar la importancia de la educar a otras personas desde la pasión, dedicación, esmero, concentración, ternura, sensibilidad y amor como educadores en el forjamiento de nuevo ciudadano, cabe destacar lo planteado por Rivas (2000):

Entender la actividad del docente como trabajo nos remite a las condiciones laborales y de producción en que tiene lugar y se aleja, por tanto, de la tradicional comprensión de la actividad docente como un servicio, una actitud de entrega (pág. 145).

Es así que, el docente hace entrega en el servicio a educar y educarse en el proceso del hecho educativo, siendo un elemento de propiciar las 
experiencias de aprendizaje en común permitiendo el valor por la dignidad humana y el mundo que rodea al estudiante, Resulta oportuno considerar, Calvo (2015a):

El profesor es un diseñador de experiencias de aprendizaje. Organiza el contenido de acuerdo al orden que logra una mayor implicación de los alumnos, negociando tiempos, modos y herramientas en un proceso puesto al servicio del desarrollo integral, del aprendizaje a lo largo de toda la vida y de la pasión por estar vivo y descubrirte a ti mismo y al mundo en la escuela. (pág. 47).

En ese mismo sentido, es importante señalar el docente de educación primaria juega un papel importante en el proceso de formación de los estudiantes relacionado al desarrollo de la innovación, creatividad entre otros aspectos que consolidan una visión holística dirigida a comprender la integridad y diversidad compleja de los seres humanos que participan en el proceso de enseñanza-aprendizaje, en otras palabras, una educación humanista y solidaria de las distintas etapas de vida de cada educando, superando los límites establecidos para concretar una educación de la hologogía como un todo desde las cosmovisión amplia de la concepción de los profesionales de la docente en el contexto de educación primaria.

\subsection{Principios de la Holística vinculada a la Praxis Docente en el contexto de Educación Primaria.}

Al hacer referencia a los Principios de la Holística, cabe destacar, Barrera (2010a): señala que la holística que se orienta en los hechos desde diversos puntos de vista, además integra todos los aspectos de las realidades, una manera de comprender la integridad de los holos, para ello planteó los principios de la holística: Principio de la Unidad del Todo, Principio de la Integralidad, Principio de la Unicidad, Principio Holográfico, Principio de Posibilidades Abiertas y Principio Sintagmático vinculados a la concepción del 
docente y su relación con los estudiantes en el contexto de educación primaria (pág. 33); como se ilustra en la figura 1.

Figura 1. Principios de la Holística y el Docente en el contexto de Educación Primaria.

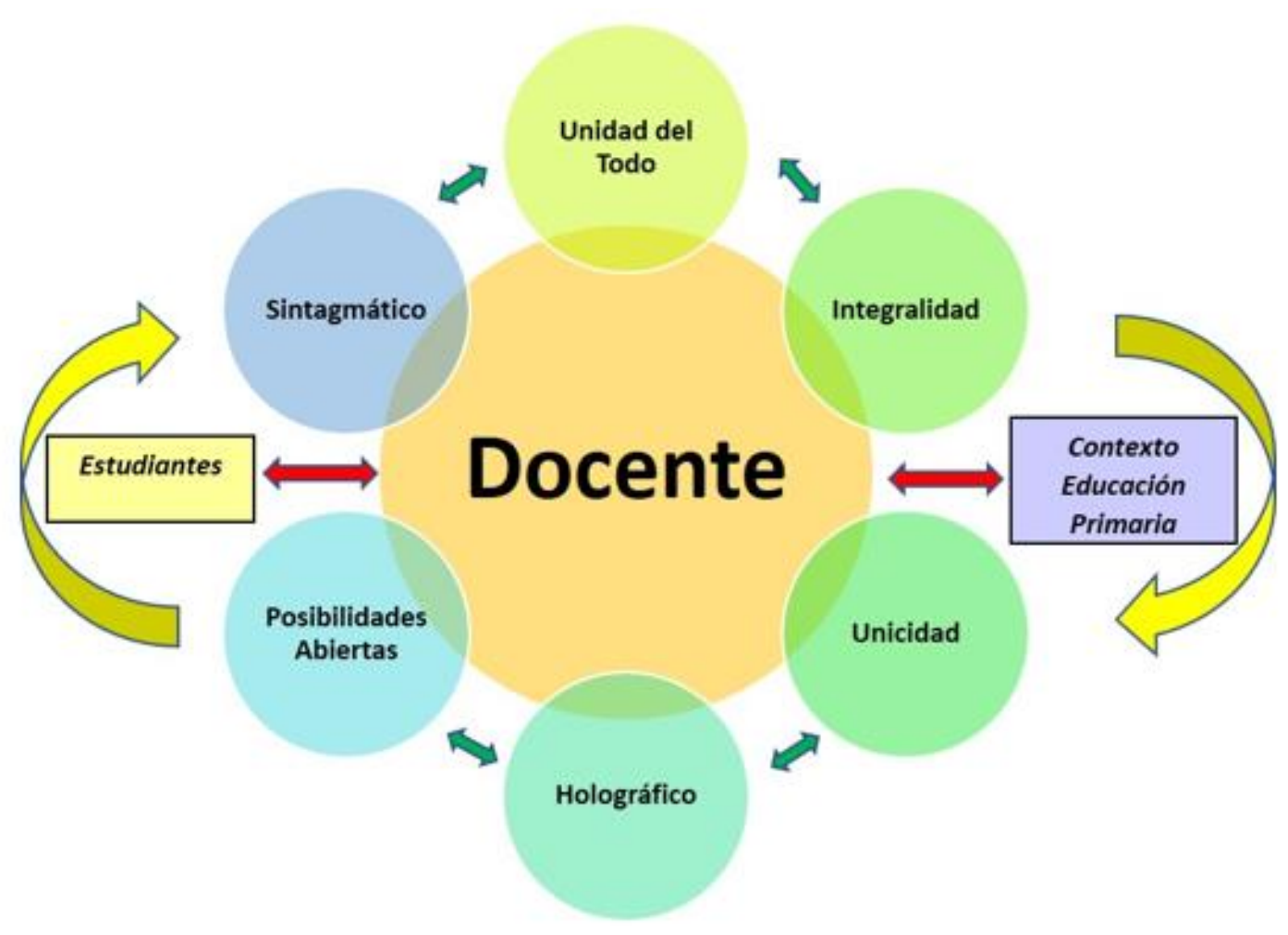

Fuente: El Autor (2019).

El docente en su concepción y visión en la labor educativa en función de aplicar los principios de la holística en el contexto de educación primaria con relación a reconocer que las realidades en el proceso educativo fluye, existiendo una continuidad permanente en las interrelaciones de los actores en las instituciones educativas, identificando la integralidad de los educandos desde su diversidad compleja en cada etapa de formación, siendo únicos como seres humanos participes en eventos y manifestaciones que expresan el todo en varios instantes de la realidad particular de persona. Además, en el 
proceso de aprendizaje-enseñanza, suelen suceder múltiples situaciones, en las cuales el docente ampliará su percepción del proceso educativo, desarrollados por lo planteado, según Barrera (2010b), en los siguientes principios: Principio de la unidad del todo, Principio de integralidad, Principio de unicidad, Principio holográfico, Principio de posibilidades abiertas, y Principio sintagmático (págs. 33-48).

\subsubsection{Principio de la unidad del todo}

La relación del principio de la unidad del todo y el docente en el contexto de educación primaria se desarrolla en cuanto, se reconoce que el proceso educativo se materializa en la formación de los estudiantes desde la unicidad de su personalidad como seres humanos en el marco de los principios de los eventos en continuidad dentro del valor circunstancial reiniciándose la realidad desde un inicio que deviene de múltiples versiones. Es allí que, la comprensión del profesional de la docencia es desde la continuidad educativa del devenir del proceso de enseñanza y aprendizaje desde la dignidad humana en el aspecto espiritual desde una metodología metafórica, metodológica y didáctica que permita una interacción docente-estudiante-escuela primaria. En conciencia, con lo planteado por Barrera (2010c):

El principio de unidad lleva a reconocerla continuidad. En un todo que deviene, que ocurre, que fluye, la precisión de límites es irrelevante y tiene valor circunstancial, a veces técnico, en oportunidades metafóricas y en otras metodológico y didáctico. Aquello que pareciera un fin se constituye en el principio de algo: toda conclusión es punto de partida. Los límites son conexiones. Las barreras constituyen fuentes de comprensión (pág. 36).

Partiendo de los anterior, el principio de la unidad del todo se relaciona con la concepción hologógica del docente, desde el reconocimiento del proceso de enseñanza y aprendizaje de los estudiantes desde su unidad como 
seres humanos que conviven con el exterior (contextos: social, educativo, emocional, entre otros) se desarrolla desde la continuidad que ocurre y confluye en las vivencias individuales de cada ser, a pesar que durante el proceso del quehacer educativo surjan barreras, que impidan el normal desenvolvimiento de las metas, el docente ante la realidad compleja se aplica la comprensión de las necesidades y requerimientos de sus educandos en la formación holística en la concreción de la dignidad humana, al respecto, Barrera (2010d):

El principio de unidad lleva a reconocerla continuidad. En un todo que deviene, que ocurre, que fluye, la precisión de límites es irrelevante y tiene valor circunstancial, a veces técnico, en oportunidades metafóricas y en otras metodológico y didáctico. Aquello que pareciera un fin se constituye en el principio de algo: toda conclusión es punto de partida. Los límites son conexiones. Las barreras constituyen fuentes de comprensión (pág. 36).

\subsubsection{Principio de integralidad}

El docente en el contexto de la educación primaria se orienta a valorar desde su praxis educativa una concepción de integralidad de los estudiantes que se expresan en distintas formas, maneras y dimensiones de sus etapas de desarrollo humano, de allí, la importancia de brindar las oportunidades en las relaciones de los profesionales de la docencia y sus educandos en el quehacer educativo en la complejidad de los seres humanos en formación, para lo cual, Barrera (2010e): "se entiende que la realidad expresa diversas dimensiones y puede ser percibida de distintas maneras, pero dicha percepción es. en oportunidades, anticipo del descubrimiento de relaciones profundas, complejas y a veces paradójicas, en contextos dinámicos" (pág. 38). 


\subsubsection{Principio de unicidad}

Desde la praxis educativa de los docentes en la educación primaria, se hace necesario vincularla con la perspectiva de reconocer la unicidad de los estudiantes desde su totalidad para comprender la diversidad dentro de las manifestaciones únicas de cada uno como ser humano que se encuentra construyendo sus saberes, conocimientos, habilidades y destrezas en la relaciones dinámicas y complejas de forma colectivo, como lo plantea, Barrera (2010f): "el pensamiento holista enfatiza la importancia de entender que la condición de totalidad se expresa de manera particular, como manifestación única e irrepetible" (pág. 39)

En consecuencia, la unicidad de los estudiantes vistos desde la labor de los docentes en las escuelas primarias surge desde la valoración e importancia de comprender la diversidad de seres humanos únicos. De acuerdo con, Barrera (2010g): "el de unicidad alude a la importancia de entender que los seres, así como los hechos, son únicos e irrepetibles" (pág. 39). Al respecto, el docente de educación primaria desde la perspectiva como diseñador de aprendizajes, experiencias y vivencias orientado al proceso de enseñanza y aprendizaje de los estudiantes desde la evaluación participativa, protagónica de cada educando, en la construcción y colaboración de los conocimientos desde lo individual y colectivo, cabe agregar, Calvo (2015b):

El profesor diseña experiencias donde expone los objetivos a alcanzar, las herramientas de evaluación y los productos para cada actividad, pero concede al alumno la autonomía de elegir su propio itinerario de aprendizaje compartiendo decisiones educativas del día a día en el aula (pág. 47).

En relación con lo anterior, la concepción hologógica del Docente vinculada al principio de la unicidad se orienta a comprender el todo desde sus múltiples realidades, desde una visión de la complejidad de las personalidades de los estudiantes en el marco de la labor del maestro dirigida al diseño de 
didácticas, estrategias y experiencias de aprendizajes relacionada con las necesidades, requerimientos de cada estudiante en su entorno social, escolar y espiritual en proceso de formación diferenciado de la etapa de vida en se encuentre en la actualidad.

\subsubsection{Principio holográfico}

El principio holográfico se relaciona con la concepción hologógica del docente en cuanto a las capacidades y posibilidades investigativas que posee en la labor educativa del contexto escolar, desde el planteamiento de Barrera (2010h): es inagotable la condición integrativa relacionada con el origen holista, siendo posible conocer la condición de la materialidad del proceso educativo y la construcción del conocimiento desde el movimiento holístico de los participantes del profeso de formación de manera continua e infinita desde un despliegue, pliegues de realidades diversas, complejas y únicas (pág. 41).

Al respecto, Barrera (2010i): "plantea que el evento contiene el todo, pues este evento, llámese hecho, situación, cosa, expresa en su constitución vestigios de una totalidad que justifica su existencia" (pág. 40). En efecto las características holográficas se orienta a la percepción de los eventos desde sus manifestaciones y rasgos que lo identifican en el marco del contenido relacional, por lo cual, se vincula con la concepción del docente de educación primaria en función de la realidad en el contexto educativo es variada, compleja, múltiple y vinculante, es preciso destacar lo planteado por Morín (1974): "la complejidad es, efectivamente, el tejido de eventos, acciones, interacciones, retroacciones, determinaciones, azares, que constituyen nuestro mundo fenoménico" (pág. 87). En este contexto, la concepción hologógica del docente se integra a los hechos, vivencias y realidades en el hecho educativo desplegado desde lo holográfico, expresa las posibilidades de la conexión infinita de los eventos desplegados siendo únicos, originales e irrepetibles relacionado con el principio de unicidad, ubicándose en el valor 
creativo del principio holográfico.

\subsubsection{Principio de posibilidades abiertas}

Al respeto del Principio de posibilidades abierta se vincula con la concepción del docente, en referencia del orden en el escolar refleja la disciplina, estructura, sistema que limita la creatividad, las posibilidades de innovar en la escuela primaria, abriendo la oportunidad del Caos se conforma como la posibilidad, apertura, creación de nuevas experiencias, vivencias, aprendizajes teniendo en cuenta el caos está inmerso en el orden, asimismo, y el orden se incluye en el caso, teniendo en cuenta que el caos sin orden sería una anarquía en los procesos. En consecuencia, Barrera (2010j):

El caos, visto como contexto dinámico de múltiples interacciones abiertas, paradójicas, evidentes y trascendentes, se confluye en matriz generadora de ideas, de experiencias, de órdenes sociales y políticos, de estructuras formales y de relaciones también transitorias. El Principio de Posibilidades Abiertas crea opciones para nuevos descubrimientos. Así como también oportunidades para comprender las cosas desde múltiples vertientes. Desde el caos, el equilibrio se ve como las posibilidades que presenta el desequilibrio; la quietud desde la turbulencia (pág. 42).

La afirmación anterior, se orienta a la consideración del caos como proceso dinámico de múltiples relaciones abiertas que se desarrollan paradójicas, evidentes y trascendentes relacionado con la labor educativa del docente de educación primario inmersa en la concepción hologógica, es identificar la fluidez en las matrices de ideas, vivencias, experiencias, ordenes sociales y políticos, de estructuras formales, relaciones humanas permanentes o transitorias, creando opciones para nuevas oportunidades y descubrimientos en función del entendimiento de los hechos, cosas o vivencias desde múltiples versiones y vertientes, manteniendo la tranquilidad desde la problemática en turbulencia, dentro de este contexto identificar las posibilidades que podrá 
estabilizar los procesos de enseñanza y aprendizaje.

\subsubsection{Principio sintagmático}

En la concepción hologógica del docente, se orienta en procesos holísticos de comprensión integral, hilado con el principio sintagmático, en función de los sintagmas definidos por Barrera (2010k): "los sintagmas son procesos de investigación, de indagación y de creación abiertos hacia interacciones permanentes, de carácter novedoso" (pág. 45). De esta manera, surge la diversas vías de conocer en el contexto de educación fundamentado con la manera sintagmática, mediante el proceso educativo de forma integral y holística en el cual se confluye conocimientos a partir de la comprensión de los actores del hecho educativo, desde la manera que ocurren los hechos en la interacción entre seres humanos, con la determinación de establecer la dinámica comprensiva que considere las posibles relaciones e identificar el sentido de las cosas en consideración a los aspectos del ser humano, de allí que Barrera (2010l), expresa que:

Alude a la comprensión integrativa del conocimiento derivada de los procesos mediante los cuales se accede a niveles de complejidad producto de procesos iterativos, como también de condiciones lógico abstractivas, y de efectos propios de la actividad perceptual. Como la realidad se presenta como un todo, el efecto de apreciación y de comprensión del todo ocurre gestálticamente, pero gracias a efectos de acercamiento a ese todo según los recursos de la integralidad se pueden efectuar "abstracciones" de la totalidad que justifican la percepción, permiten la orientación y facilitan el conocimiento (pág. 43).

Por todo lo dicho, en la concepción hologógica del docente relacionadas con los planteamientos de Barrera se vinculan estrechamente con la metáfora filosófica del sintagma como consecuencia de la sincronía y de simultaneidad de los hechos y realidades que convergen en el contexto de educación primaria en el marco de la diversidad de aspectos, con característica dinámico 
vinculado con el principio de integralidad.

\section{Reflexiones Finales}

Al respecto, desde la concepción y praxis de los docentes en la educación primaria y los principios de la holística son elementos importantes para que surja un proceso educativo integral en el reconocimiento de la diversidad en la realidad compleja de los estudiantes en función del reconocimiento como seres humanos, en garantía de un hecho educativo humanista, espiritual con la generación de conocimientos de manera colectiva desde la individualidad.

Finalmente, se requiere en el contexto de educación primaria, desde la practica educativa del docente procesar en el esquema de los procesos de enseñar, educar y generación de conocimientos, destrezas, habilidades en los estudiantes que le sean de interés para su formación en el marco de los Principios de la Holística, de tal manera que desde la importancia que les asigne, significará un aprendizaje valioso para sus vidas, de allí que el maestro oriente su acción a propiciar espacios de construcción e intercambio de aprendizaje mediante las estrategias que motive y anime a los participantes del hecho educativo, cumpliendo así un proceso de desarrollo de los aprendizajes desde su significado e importancia, donde interviene el docente como mediador del proceso de enseñanza y aprendizaje holístico.

\section{Referencias}

Argyris, Ch., \& Schön D. (1978). Organizational Learning: A Theory of Action Perspective. Chicago, EE. UU.: Publisher Dryden Press.

Barrera, M. (2010a,b,c,d,e,f,g,h,i,j,k,l). Holística. Segunda Edición, Caracas, Venezuela: Ediciones Quirón.

Camacaro, Y. (2017). Los Valores del Docente Coligados a la Ética y Postmodernidad. Revista Scientific, 2(3), 362-379, e-ISSN: 2542- 
2987. Recuperado de: https://doi.org/10.29394/scientific.issn.25422987.2017.2.3.20.362-379

Calvo, A. (2015a,b). Viaje a la Escuela del Siglo XXI: Así trabajan los colegios más innovadores del mundo. Depósito Legal: M-301072015, ISBN: 978-84-15282-14-3. Madrid, España: Ediciones Fundación Telefónica.

Delors, J. (1996). La Educación encierra un Tesoro. Informe a la UNESCO de la Comisión Internacional sobre la educación para el siglo XXI. París, Francia: Ediciones UNESCO. Recuperado de:

http://www.unesco.org/education/pdf/DELORS S.PDF

Freire, P. (1977a,b,c). La Educación como práctica de la libertad. Biblioteca Clásica del Siglo Veintiuno, 3ra edición, 1ra reimpresión. Buenos Aires, Argentina: Siglo XXI Editores.

Freire, P. (1993a,b,c). Cartas a quien pretende enseñar. Biblioteca Clásica del Siglo Veintiuno, 2da edición, 3ra reimpresión. Buenos Aires, Argentina: Siglo XXI Editores.

Informe Nacional de la República Bolivariana de Venezuela (2004a,b). Políticas, Programas y Estrategias de la Educación venezolana. Caracas, Venezuela: Ministerio de Educación y Deportes. [Documento en línea]. Recuperado de:

https://www.oei.es/historico/quipu/venezuela/Pol Estrategias Educ.pdf McLaren, P. (2005a,b). La vida en las escuelas, una introducción a la pedagogía crítica en los fundamentos de la educación. Coyoacán, México: Siglo XXI editores, S.A. de C.A.

Morín, E. (1974). El paradigma perdido. Barcelona, España: Editorial Kairós. UNESCO (2016). La Educación Transforma vidas. París, Francia: Organización de las Naciones Unidas para la Educación, Ciencia y Tecnología. Recuperado de:

https://es.unesco.org/themes/\%C3\%A9ducation 
UNESCO (2015a,b). Declaración de Incheon: Educación 2030: Hacia una Educación Inclusiva y Equitativa de Calidad y un Aprendizaje a lo Largo de la Vida para Todos. ED/WEF2015/MD/3. Incheon, Korea: World Education Forum. Recuperado de:

https://unesdoc.unesco.org/ark:/48223/pf0000233137 spa

Rivas, J. (2000). Profesorado y reforma, ¿un cambio de las prácticas de los docentes? ISBN: 84-95212-74-9. Málaga, España: Ediciones Aljibe. 


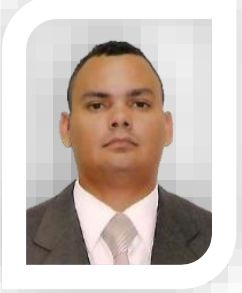

Nací en La Guaira, estado Vargas, Venezuela, el 7 de mayo del año 1985. Licenciado en Educación de la Universidad Nacional Experimental Simón Rodríguez (UNESR); Magister Scientiarum en Educación Superior de la Universidad Nacional Experimental de la Fuerza Armada Bolivariana (UNEFA); Doctorante en Ciencias de la Educación de la Universidad Latinoamericana y del Caribe (ULAC); Participante de la carrera de Derecho en la Universidad Bicentenaria de Aragua (UBA); Comisionado Municipal de la Oficina Antidrogas del municipio Independencia; Director de Recursos Humanos de la Alcaldía del municipio Independencia; Jefe de Personal de la Policía Municipal Independencia; Director de Administración, Finanzas y Presupuesto de la Contraloría del municipio Simón Bolívar; Tutor de Trabajos Especiales de Grado (Especializaciones y Maestrías) en la Universidad Pedagógica Experimental Libertador (UPEL) y la Universidad Nacional Experimental de la Fuerza Armada Bolivariana (UNEFA); Docente de Educación Media General en la Unidad Educativa Nacional 28 de Marzo; Coordinador de Control de Estudios de la Unidad Educativa Privada Coronel Antonio Nicolas Briceño; Docente de Educación Primaria de la Unidad Educativa Nacional Bolivariana Guaicaipuro. Arbitro Calificado de la Revista Scientific (e-ISSN: 2542-2987). 